\title{
Mechanical Effects of Solid Water on the Particle Skeleton of Soil: Mechanism Analysis
}

\author{
Xiaomeng Duan $\mathbb{D D}^{1,2}$ \\ ${ }^{1}$ Institute of Geotechnical Engineering, Yango University, Fuzhou 350015, China \\ ${ }^{2}$ College of Civil Engineering, Yango University, Fuzhou 350015, China \\ Correspondence should be addressed to Xiaomeng Duan; xmd2132@126.com
}

Received 4 March 2021; Accepted 17 May 2021; Published 2 June 2021

Academic Editor: Giovanni Mongelli

Copyright (C) 2021 Xiaomeng Duan. This is an open access article distributed under the Creative Commons Attribution License, which permits unrestricted use, distribution, and reproduction in any medium, provided the original work is properly cited.

\begin{abstract}
It is generally accepted that the adsorbed water layer on the surface of the mineral particle has significant effects on the mechanical properties of soils. By defining the concepts of "solid water" and "particle skeleton" after a brief review on adsorbed water, therefore, the mechanical mechanism about how solid water affects the deformation and strength of particle skeleton is theoretically clarified, which could be the physical basis of the reasonability of two assumptive conditions for effective stress equation. Considering solid water as a two-dimensional liquid with appreciable normal strength and lubricity, if soil particles are always wrapped by solid-water layer, the only mechanical effect due to water pressure is to compress particles; while if the interparticle solid water could be extruded undergoing enough force with suitable confinement, the mechanical effects due to increasing water pressure are not only to compress particles more but also to enhance interparticle friction because the indirect interparticle contact could be changed into direct contact to consequently alter the interparticle friction. Because solid water is not likely to be extruded by pressure alone, if the particle compression is negligible relative to the soil-mass compression, two assumptive conditions for effective stress equation are reasonable. Moreover, a simple monitoring test on water content is conducted to certify that the solid-water layer should always exist in soils under ambient conditions, so the ordinarily oven-dried soil samples used in conventional geotechnical tests carried out under ambient conditions could be just "nominally dry" samples with the effects due to solid water.
\end{abstract}

\section{Introduction}

Even under ordinary circumstances, water and soil particles interact with each other because water molecules are strongly attracted to and adsorbed on soil particle surfaces, particularly to clays at low water contents (low saturation); the water structure can be quite different than that in wet or saturated clays [1]. It is a consensus that the existence of adsorbed water film is significant for unsaturated porous media, which is associated with hydraulic conductivities [2], matric potential (suction) [3,4], water retention [5-7], strength [8-10], and deformation [11-13] of soils. However, because of diverse comprehension on the complicated physical and chemical water-mineral interactions, the diversity of water properties is not involved enough in soil mechanics, while "contractile skin" is considered an independent phase in
Fredlund's theory of stress state variable $[14,15]$ and adsorption is considered in some works on effective stress $[16,17]$. In fact, the mechanical mechanism about how adsorbed water affects the properties of soil skeleton is not totally clear yet. Such as, for the keystone of soil mechanics, stress framework, the reasonability of the principle of effective stress should be associated with the existence of adsorbed water.

Initially, according to the test phenomena, Terzaghi [18] concluded that the neutral stress produces neither a measurable compression nor a measurable increase of the shearing resistance. Then, Bishop and Blight [19] concluded that two conditions are necessary and sufficient for effective stress equation to hold rigorously for both volume change and shear strength if the conditions are put in the following form:

(1) The soil particles are incompressible 
(2) The yield stress in the particle material, which controls the contact area and intergranular shearing resistance, is independent of the confining pressure

However, for two contacted particles undergoing only homogeneous water pressure as shown in Figure 1(a), it is interesting that the water pressure should cause the interparticle pressure at the contact point as shown in Figure 1(c), which may, theoretically, induce higher friction strength to resist the interparticle shear movement when the water pressure is increased. If the particles are yieldable, the detailed interparticle contact zone is shown in Figure 1(b), and for particle I, three different stress/pressure concepts with the same numerical value are illustrated with different colors in Figure 1(d): real water pressure, inner-particle stress, and interparticle pressure. Because the shear strength at the contact is assumed to be as in Equation (1) [20], for the particles in Figure 1, the shear strength due to water pressure at the contact, $\tau_{\mathrm{s}}{ }^{\mathrm{WP}}$, should be as in Equation (2), where $u_{\mathrm{w}}$ is numerically equal to the water pressure but its real physical meaning is the interparticle pressure due to water pressure.

$$
\begin{aligned}
\tau_{\mathrm{s}} & =c_{\mathrm{s}}+\sigma_{\mathrm{s}} \cdot \tan \phi_{\mathrm{s}}, \\
\tau_{\mathrm{s}}^{\mathrm{WP}} & =c_{\mathrm{s}}+u_{\mathrm{w}} \cdot \tan \phi_{\mathrm{s}},
\end{aligned}
$$

According to Equation (2), it is clear that $\tau_{\mathrm{s}}{ }^{\mathrm{WP}}$ should be increased with the increasing $u_{\mathrm{w}}$, which is contradictory to the test results concluded by Terzaghi [18].

Evidently, based on the two-particle system as shown in Figure 1, there should be two theoretical effects on soil particles due to water pressure: (1) particle compression and (2) interparticle friction, but both are contradictory to Terzaghi's test results. So, the questions are whether the two assumptive conditions for effective stress equation are reliable although they are based on test phenomena and what reason causes the contradiction between test and theoretical analysis in Figure 1. One purpose of the present work is to find out the answers.

Firstly, the properties of adsorbed water are briefly reviewed and then the terms "solid water" and "particle skeleton" are defined based on a six-phase model of soils. Then, it is focused on the potential effects of solid water on the strength and deformation of particle skeleton, that is, to clarify the mechanism about how solid water affects the mechanical properties of particle skeleton, furthermore to illustrate the reasonability of the hypotheses in the principle of effective stress. Finally, the influence of solid-water layer on common geotechnical tests is discussed based on some data and phenomena.

\section{The Definition and Properties of Solid Water}

Soils adsorb water molecules from air and retain them physically combined even in air medium [21]. Low [22] concluded that water molecules adjacent to a clay mineral surface could be bonded to both the oxygen atoms and the hydroxylic groups of the surface by covalent hydrogen bonds. Based on numerous research results, it is a complete unanimity that "water adsorbed on clay surfaces differs in structure and physical properties from bulk liquid water", so two representative models of adsorbed water has been summarized as (1) a solid-like (or ice-like) substance or (2) a two-dimensional fluid [23]. Early concepts of the structure of adsorbed water suggested an ice-like character because of the similarity between the hexagonal symmetry of the oxygens and hydroxyls in clay surfaces and the structure of ice, but subsequent studies have shown that the structure cannot be that of ice $[1,22,23]$. However, the term "ice-like" is still used in recent works because of a strongly coordinated structure of adsorbed water [24-26].

The main difference between these two models is the shear strength. That is, based on the solid model, adsorbed water could resist both normal and shear forces to a larger degree than liquid water, but the two-dimensional fluid could resist appreciable normal stress but would shear perhaps even more easily than normal liquid water because the number of bonds that can form between the polar water molecules is considerably reduced from what it would be in normal liquid water due to the surface constraint. Nevertheless, for both models, the normal strength of adsorbed water is stronger than in liquid water because of the constraint due to the clay surface [23]. It means that the adsorbed water, which has a modulus between the moduli of bulk water and solid particles [17], could be strong enough to bear and transmit loads with soil skeleton together.

Based on the analysis of numerous data, the model of twodimensional liquid is recommended [23]. Considering the existence of hydration force $[27,28]$ and lubrication $[26,28]$ due to adsorbed water, it seems that the two-dimensional liquid model is more reliable than the solid-like model.

Because the ordered hydration layer is strongly attached and resists being removed or squeezed-out by compression, there is a strong short-range repulsive force that acts between polar surfaces separated by a thin layer $(<3 \mathrm{~nm})$ of water, named hydration force which has been observed for clays, minerals, and even glasses [27-30]. The hydration layers could resist a large normal load to prevent approach of the surfaces, but at same time, they remain in a very fluid manner with a viscosity much closer to the bulk viscosity under shear, so the hydration layers act as highly efficient lubricants [31]. It is demonstrated that the interparticle shear strength inversely depends on the number of water layers between two particles [32]. Morrow et al. [33] have examined 15 gouge minerals to demonstrate their frictional strengths are related to the amount of adsorbed water, and it is clear that many minerals show a decreased tendency in frictional strength after saturation. In conclusion, the two surfaces may slide mutually with a very low friction due to the low shear strength of water [28].

Based on the above review, it is clear that adsorbed water is so significant in geotechnical engineering due to some reasons as follows:

(1) Adsorbed water should widely exist in geomaterials under ambient conditions

(2) Adsorbed water should be strongly attached and highly ordered, which could be strong enough to bear and transmit loads with soil skeleton together

(3) Adsorbed water could lubricate the interparticle friction 


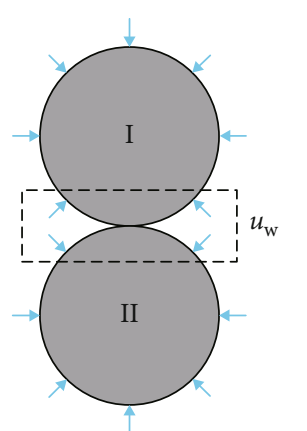

(a)

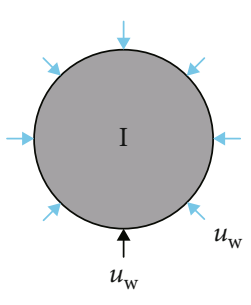

(c)

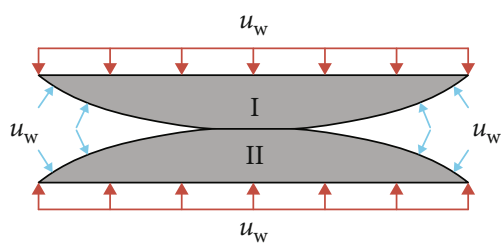

(b)

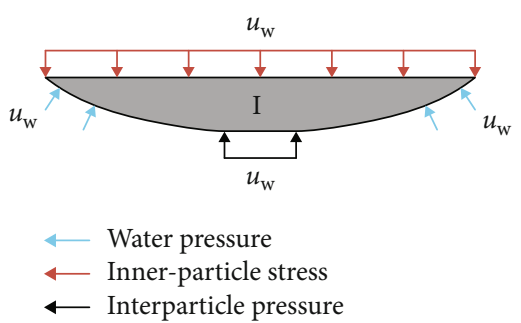

(d)

Figure 1: Illustration of two contacted particles: (a) two contacted particles undergoing homogeneous water pressure, (b) detailed equilibrium of the contact zone, (c) interparticle pressure on the bottom of particle I, and (d) water pressure, inner-particle stress, and interparticle pressure on the contact zone of particle I.

Because the adsorbed water could be defined as "the entire interfacial region between the oxygen or hydroxyl surface of the clay crystal and bulk liquid water" [23], traditionally, the water in soils are briefly classified as adsorbed water and bulk water, or named bound water and free water. More precisely, the water in soils could be classified as (1) adsorption water, also called hygroscopic water; (2) viscous water, or capillary water; and (3) free water [34]. Moreover, the contract skin could be considered an independent phase in soils [14].

In our recent work [35], a six-phase model for unsaturated soil is proposed, as shown in Figure 2. That is, unsaturated soil is divided into six independent phases: soil particle, cement, solid water, liquid water, contractile skin, and pore air. The part of pore water, which behaves as semisolid or solid and could bear and transmit loads with soil skeleton together, is considered an independent phase named solid water which is the part of adsorbed water strongly adsorbed on the surfaces of soil particles. In contrast, the rest of pore water, which still behaves as liquid, is named liquid water as an independent phase. It is worth to note that there is no explicit boundary but a transitional zone between the solid and liquid water. However, this classification is beneficial to analyze relevant issues with simplicity.

\section{Effects of Water Pressure on Particle Skeleton considering the Existence of Solid- Water Layer}

Based on the six-phase system, it is easy to know that the essence of soil deformation is the movement and deforma- tion of soil particles, so the assembly of soil particles is defined as particle skeleton which is the core of the bearing structure of soil. As mentioned above, adsorbed water should widely exist in geomaterials under ambient conditions, so soil particles are usually wrapped by the solid-water layer, as shown in Figure 2(a). Therefore, in this section, the mechanical mechanism about how the solid-water layer influence the effects of water pressure on the strength and deformation of particle skeleton is analyzed based on simplified models.

3.1. The Compression of Soil Particles due to Water Pressure. The first potential effect of water pressure on particles is to compress them, except for a "perfect" solid which is defined with the angle of intrinsic friction, $\psi=0$, and the compressibility, $C_{s}=0$, i.e., a "perfect" solid is incompressible. However, real soil particles have a finite compressibility, $C_{s}$, so an increment of hydrostatic pressure, $\Delta u$, will produce a volume decrement by an amount as in Equation (3) [20].

$$
-\left(\frac{\Delta V}{V}\right)_{\mathrm{s}}=C_{\mathrm{s}} \cdot \Delta u
$$

Some representative values of $C_{\mathrm{s}}$ are also given by Skempton [20], such as for sand, $C_{\mathrm{s}}=2.7$, and for London clay, $C_{s}=2.0$. However, when the compressibility of soil mass, $C$, is taken into consideration, it will at once be seen that for soils the ration $C_{s} / C$ is negligible, which is believed to be the reason that Terzaghi's equation is acceptable to a high degree of approximation [20]. But, for the case that particles are very "soft" with considerable $C_{\mathrm{s}}$ and the increment of $\Delta u$ is very large, a nonnegligible particle compression should be considered. Therefore, if the concept of effective 


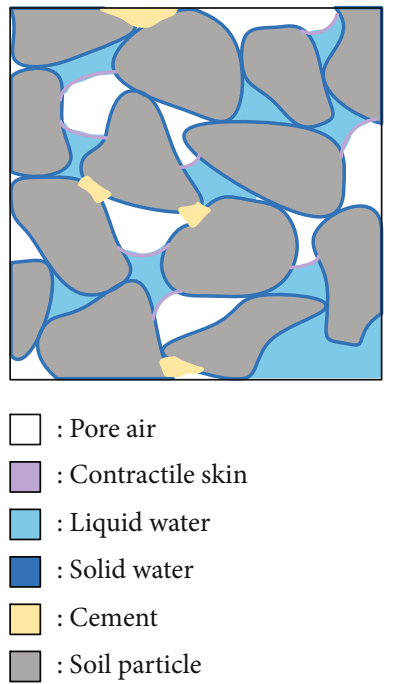

(a)

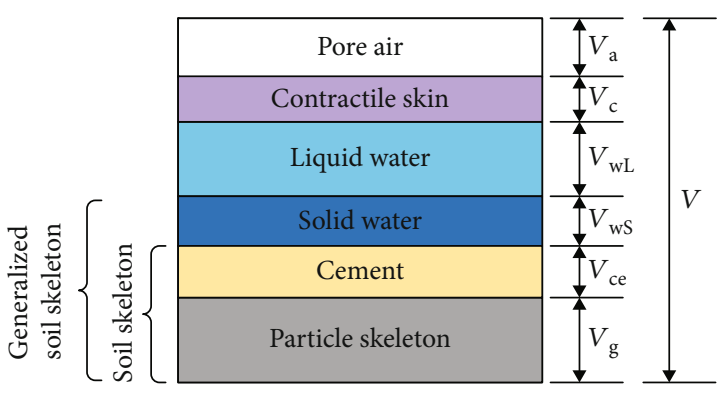

(b)

FIGURE 2: Illustration of the six-phase model for unsaturated soil: (a) the six-phase representative volume element (RVE) and (b) volumetric illustration [35].

stress is defined as "the stress controlling the strength and deformation of soil exclusively," the assumption that "soil particles are incompressible" is necessary for the principle of effective stress to hold rigorously for volume change.

For the theoretically smooth particles (neglecting the roughness and microcracks of particles) undergoing only water pressure, if they are directly contacted, i.e., there is no interparticle solid-water layer, as shown in Figure 1(a), the equilibrium of contacted particles is similar to the hydrostatic equilibrium of particles suspended in water, but just the physical significance of the interparticle pressure is different to the water pressure although they have the same numerical value, as shown in Figure 1(c). When considering the existence of solid water, as shown in Figure 3(a), because the solid-water layer could transmit water pressure, the particle is similarly suspended in water and its equilibrium state is also in hydrostatic state as shown in Figure 3(b). Even if the interparticle solid-water layer could be totally extruded, the surrounded pressure state due to water pressure is still similar to the hydrostatic state but with different physical meanings of pressure, as shown in Figure 4(d). Therefore, apparently the existence of solid-water layer could not affect the particle compression due to water pressure because whether the solid-water layer exists or not, the equilibrium of the particle is always similar to the hydrostatic equilibrium. However, if the roughness and microcracks of particle are considerable, for the case without interparticle solidwater layer (direct particle-particle contact), it should be impossible to transmit water pressure equally to everywhere at the contact zone and into every microcrack, so increasing water pressure might induce inhomogeneous deformation at the contact zone, and the solid-water layer in microcracks is beneficial to the particle crushing under high water pressure. But if the solid-water layer always exists, the equilibrium of the particle due to water pressure should always be the hydrostatic state. In a word, the existence of the solid- water layer is conducive to keep the hydrostatic equilibrium of particles.

In conclusion, for a soil mass undergoing only water pressure, the existence of the solid-water layer makes soil particles similarly suspended in water to keep their hydrostatic equilibrium, so that "the soil particles are incompressible" could be the only precondition to make that "the neutral stress does not produce a measurable compression" reliable. If the interparticle solid-water layer could be extruded to realize the direct particle-particle contact, the roughness and microcracks of particles could be the reasons for the inhomogeneous deformation and crushing of particles under high water pressure. However, the solid water is not likely to be extruded by pressure alone but enough forces with suitable confinement are needed (as discussed in the next subsection), so the "suspended case" of particles is more reasonable for a soil mass undergoing only water pressure.

3.2. The Interparticle Force due to Water Pressure. For the interparticle friction, although the shear strength due to water pressure could be calculated by Equation (2) based on the two-particle model in Figure 1, the particles in natural soils may not contact directly because of the solid water [36] which wraps soil particle and is strong enough (with high viscosity) to transmit interparticle force and meanwhile water pressure. It means that if the solid-water layer exists, a force chain in soil is in the configuration of "particle-solid waterparticle." Because it is concluded above that the solid water should widely exist in geomaterials under ambient conditions, in this subsection, the effect of water pressure on the interparticle friction is analyzed in consideration of the properties of solid water.

Firstly, a simple thought experiment is taken to illustrate the effect of the existence of fluid on the contact property. Imagine a small water tank that contains a solid cube (the water pressure is assumed to be constant and the gravity is 


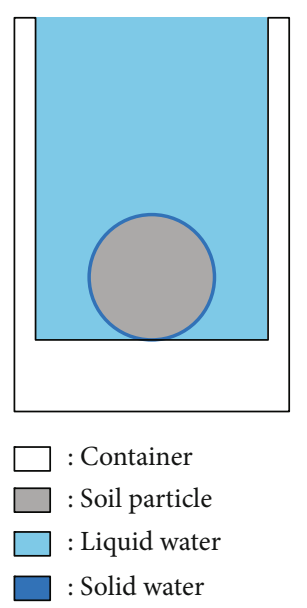

(a)

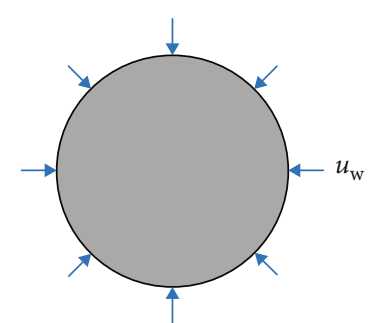

Water pressure transmitted by solid water

FIGURE 3: Illustration of a single soil particle-tank system without load and gravity but constant water pressure: (a) single soil particle with solid-water layer in water tank and (b) the water pressure transmitted by solid water onto the particle.

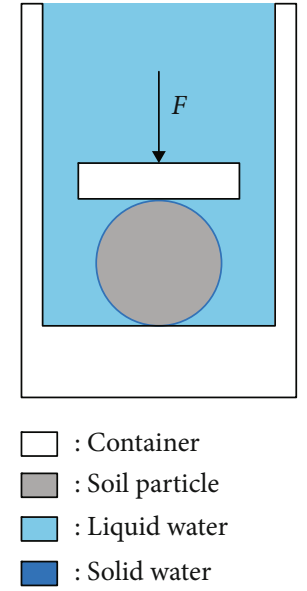

(a)

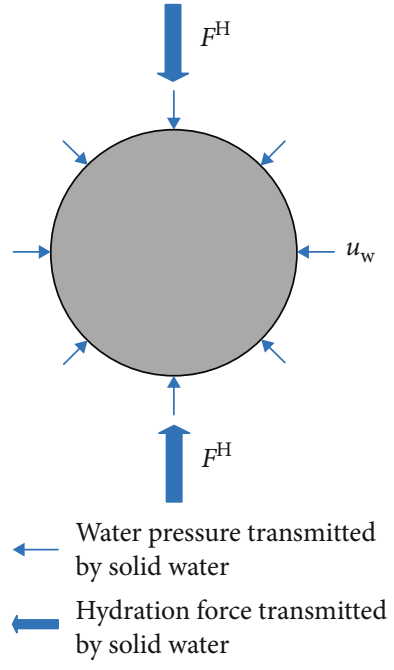

(b)

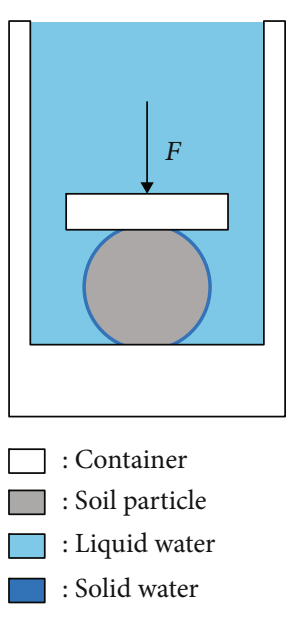

(c)

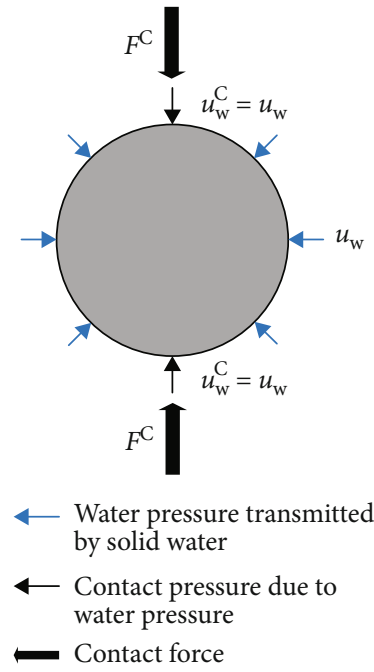

(d)

FIGURE 4: Illustration of a single soil particle-tank system with constant water pressure and nongravity: (a) the water tank with a soil particle with solid-water layer (indirect contact), (b) the water pressure and hydration force transmitted by solid water onto the particle surface, (c) the water tank with a soil particle without interface solid-water layer (direct contact), and (d) the water pressure, contact pressure, and contact force on particle surface.

neglected), as shown in Figure 5(a). No matter how close the cube approaches the tank bottom, so long as there is water between the contact surfaces, the pressure on every surface of the cube is real water pressure, $u_{\mathrm{w}}$, as shown in Figure 5(b). It means that the cube is suspended in water, so there is no friction force between the cube and the tank bottom because there is just water cube contact but no tank-cube contact. When the cube is actuated by the external force $F$ to move in the tank, the total resistance is only due to the water viscosity (Figure 5(a)).

If the cube directly contacts with the bottom (the water between the contact surfaces is squeezed out thoroughly) as in Figure 5(c), there is no water pressure acting on the bottom of the cube but a contact pressure equal to water pressure, $u_{w}^{\mathrm{C}}$, which is induced by the water pressure on the cube top, as shown in Figure 5(d). In this case, when the cube moves, the total resistance is derived from not only the water viscosity but also the bottom friction (Figure 5(c)). In conclusion, the water pressure can cause contact friction only if two parts contact directly without interface water.

An interesting practical application based on the mechanism in Figure 5 is the suction cup. If a suction cup is naturally put on a wall surface, as shown in Figure 6(a), there is no suction between the cup and wall because the air pressure is balanced on both sides of the cup (Figure 6(b)). When the air between the cup and wall is squeezed out, i.e., the cup directly contacts with the wall surface as shown in Figure 6(c), it is "sucked" on the wall. In this case, although 


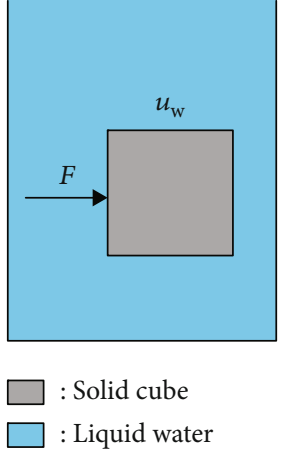

(a)

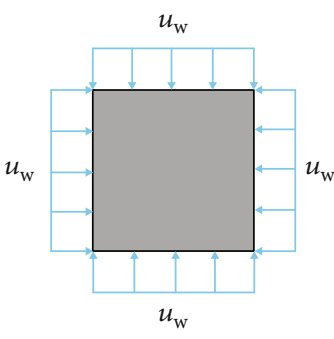

(b)

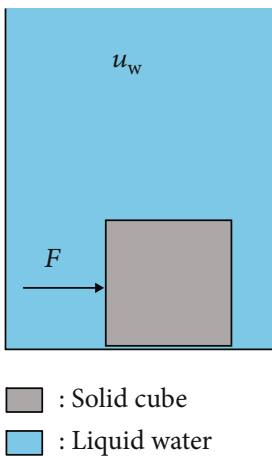

(c)

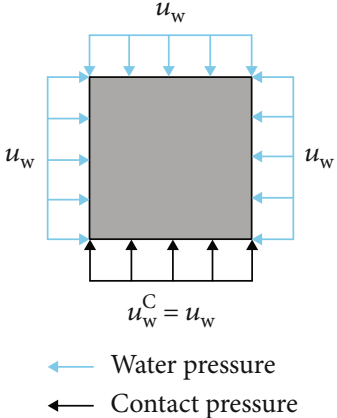

(d)

FIGURE 5: Illustration of a cube-tank system with constant water pressure and nongravity: (a) the water tank with a suspended cube (water-cube contact), (b) the water pressure on the suspended cube surface, (c) the water tank with a contacted cube (tank-cube contact), and (d) the water and contact pressures on the contacted cube surface.

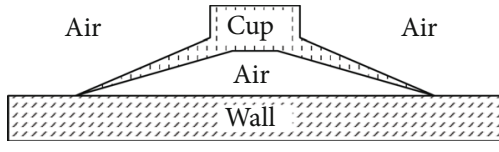

(a)

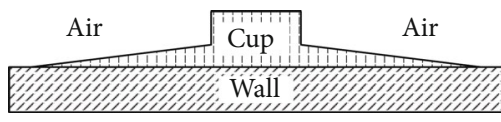

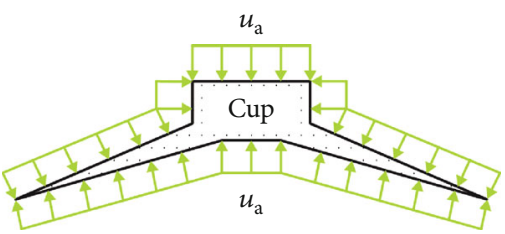

(b)

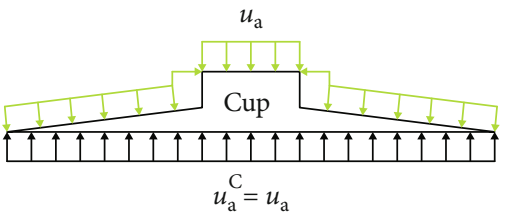

$\longleftarrow$ Air pressure

$\longleftarrow$ Contact pressure

(d)

FIGURE 6: Illustration of a suction cup-wall system with constant air pressure and nongravity: (a) the naturally contacted cup-wall system, (b) the air pressure on the cup, (c) the "sucked" cup-wall system, and (d) the air and contact pressures on the cup.

the pressures on both sides of the cup are still balanced, the pressure on the top is real air pressure, $u_{\mathrm{a}}$, while the other on the bottom is the contact pressure due to (and equal to) the air pressure, $u^{\mathrm{C}}$, as shown in Figure 6(d). In fact, the cup is "pushed" on the wall by the air pressure but not "sucked." Because of the direct cup-wall contact, there should be considerable interface friction; moreover, the friction could be lubricated if an interface water layer exists between the cup and wall.

Based on the above discussion, it is clear that the contact condition (direct or indirect) determines the friction state, even though the equilibrium of a single part could be similar. So, for the soils with solid water, the solid-water layer could affect the interparticle contact condition to consequently alter the interparticle friction.

Similarly, imagining a tiny water tank contains a soil particle (the water pressure is assumed to be constant and the gravity is neglected), as shown in Figure 4(a). Initially, the soil particle is wrapped by solid water; therefore, the particle contacts the tank bottom indirectly, as well as in suspension. If an increasing force $F$ acts on the particle, the solid water will be squeezed out gradually. Because the solid-water layer could offer a considerable hydration force, the load, $F$, is transmitted though the solid water by the hydration force, $F^{\mathrm{H}}$, onto the particle surface; simultaneously, the water pressure is still transmitted by the solid-water layer onto the particle surface. Therefore, the equilibrium of the particle in Figure 4(a) is shown in Figure 4(b). Until the load is larger than the maximum value of hydration force that the solidwater layer could offer, the interface solid-water layer will be squeezed out, i.e., the direct contact is achieved, as shown in Figure 4(c). In this case, the water pressure can cause the corresponding contact pressure and the load is directly transmitted. Therefore, the equilibrium of the particle in Figure 4(c) is shown in Figure 4(d), which is similar to Figure 4(b) but has different force/pressure components.

Conclusively, because of the wide existence of solid water under ambient conditions, soil particles are similarly 

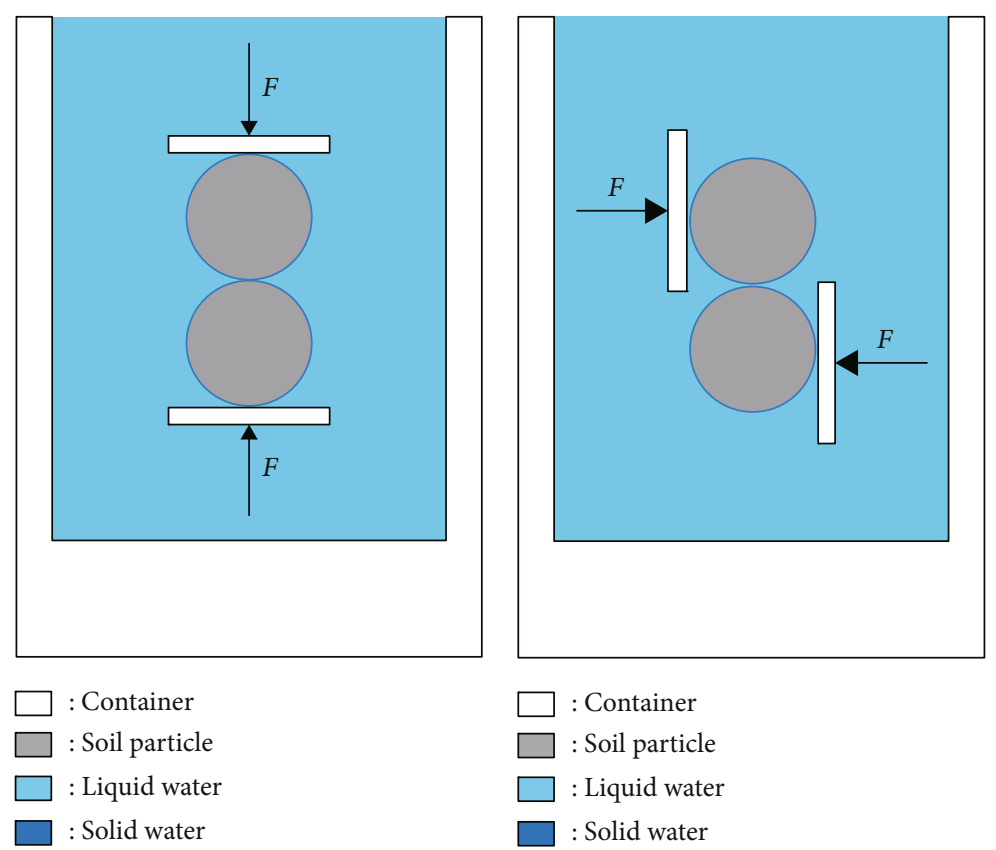

(a)

(b)

FIGURE 7: Illustration of two directions of interparticle movement affected by the solid-water layer: (a) the approaching movement resisted by repulsive hydration force and (b) the lubricated slip/shear movement.

suspended in pore water; so that the water pressure could not cause interparticle contact pressure in the case of indirect contact. If only particles can continually access each other until the interparticle solid-water layer is completely squeezed out, the interparticle pressure due to water pressure would arise. Nevertheless, pressure alone is not likely to be sufficient to squeeze out all the water between parallel particle surfaces in naturally occurring clays [1]. Moreover, the considerable repulsive hydration force could resist the extrusion of interface solid water, but based on the two-dimensional fluid model, the solid-water layer could also lubricate the interparticle friction. It means that the interparticle solidwater layer could resist the approaching movement in Figure 7 (a) but facilitate the slip/shear movement in Figure $7(b)$. Therefore, for a particle skeleton, the confinement condition could be important to determine whether the interparticle solid-water layer could be extruded or not.

In other words, for a particle skeleton, because of the strength of solid water, it could be difficult to squeeze out the interparticle solid-water layer if the particle movement is not rigidly restricted: the skeleton may restructure spontaneously when some particles approach each other to a certain extent. Imaging a particle skeleton of three particles as shown in Figure 8(a), initially, the particles are wrapped by solid water, i.e., they contact the bottom and each other indirectly. If an external force $F$ is acted on the particle skeleton, the particles will get closer gradually, and then, the approaching movement, consequently, will be resisted by the repulsive hydration force. In addition, the lateral movement of bottom particles is not confined rigidly and the interparticle friction is lubricated by the solid water, so they may spontaneously move laterally to avoid the squeezing out of solid water, i.e., particles would prefer to slip away rather than get closer.
Then, the particle skeleton may restructure to the configuration as shown in Figure 8(b). Up till now, the particles cannot move continually; thus, the interface solid water will be squeezed out gradually with the increasing of $F$. Finally, the particles contact with the tank bottom directly as shown in Figure 8(c).

If the particle movement is confined rigidly, the skeleton cannot restructure freely. Consequently, the particles can only get closer and closer until the interface solid water is totally squeezed out. Imaging the particle skeleton with lateral restriction as shown in Figure 9(a), with the increasing of external load $F$, the interface solid water will be squeezed out gradually until the particles contact the bottom and each other directly, as shown in Figure 9(b).

In conclusion, because the solid water could resist a compression load but not a shear load, i.e., it could resist the approaching movement but facilitate the slip/shear movement, if the particle movement is not rigidly confined, the interparticle direct contact (true particle-particle contact) as shown in Figure 1 may not present in natural soils because of the wide existence and difficult extrusion of solid water.

In fact, for a real particle skeleton of soil, the problem is more complicated than the above models because the water pressure is not constant and the gravity should be taken into consideration. For a soil mass without external load, the equilibrium of single particle is associated with the gravity and buoyancy. If the difference between gravity and buoyancy of a particle is not large enough to extrude the interparticle solid-water layer, the particle just directly contact with (wrapped by) solid water, i.e., it is similarly suspended in water. This case should be suitable for the soils with smaller particle size, especially for silts and clays. Otherwise, if the difference between gravity and buoyancy of a particle is large 

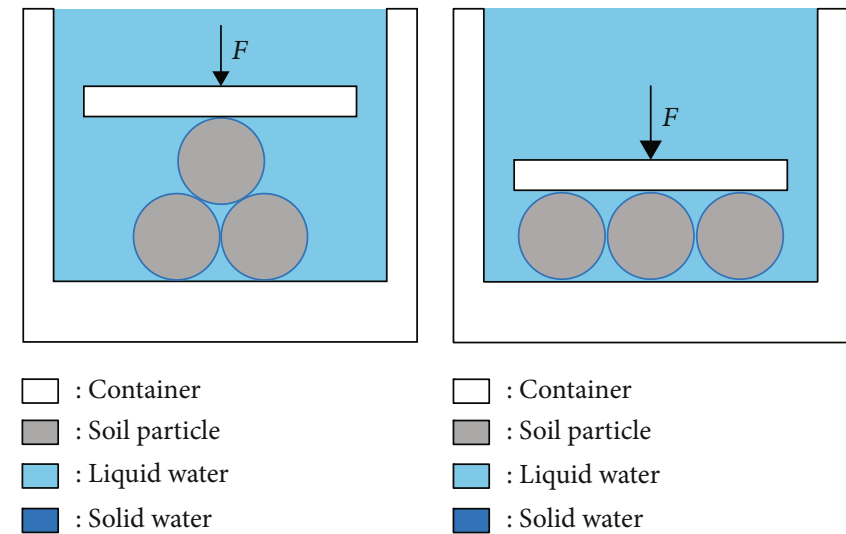

(a)

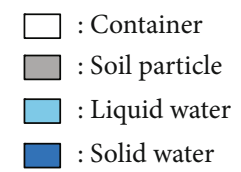

(b)

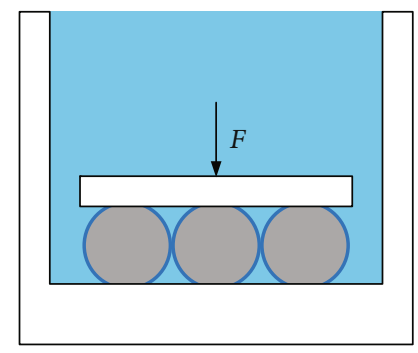

Container

Soil particle

: Liquid water

: Solid water

(c)

FIGURE 8: Illustration of a particle skeleton with solid water bearing external force without lateral restriction: (a) initially indirect contact, (b) indirect contact after restructuring, and (c) finally, direct contact.

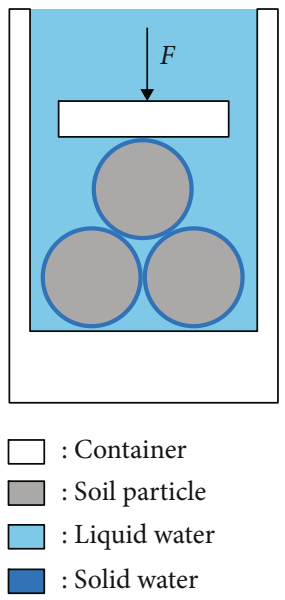

(a)

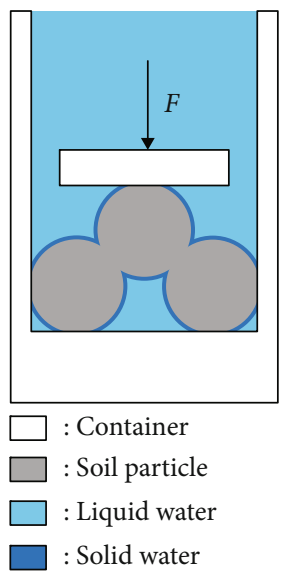

(b)
FIGURE 9: Illustration of a particle skeleton with solid water bearing external force with lateral restriction: (a) initially indirect contact and (b) finally, direct contact.

enough, i.e., it is larger than the maximum value of hydration force, the interparticle solid-water layer could be totally extruded to induce the direct particle-particle contact. This case should be suitable for the soils with larger particle size, especially for gravels.

In summary, when the solid-water layer always exists, soil particles are similarly suspended in water while the only mechanical effect due to water pressure is to compress particles. If the intrinsic compressibility of particles is considerable, large pore pressure could induce evident volume compression, but for most soils, the particle compression is negligible relative to the soil-mass compression. When the interparticle solid water could be removed under appropriate conditions, such as enough force (self-weight and external loads) with suitable confinement to extrude, or high temperature to evaporate, the indirect interparticle contact (the configuration of "particle-solid water-particle", named "lubricated contact") could be changed into the direct interparticle contact (the configuration of "particle-particle", named "friction contact"). It means that the interparticle friction property is changed due to the extrusion of the interparticle solid-water layer. In this case, the mechanical effects due to the increasing water pressure are not only to compress particles but also to enhance the interparticle stiction/friction.

For a natural soil mass without external load, pressure alone is not likely to be sufficient to squeeze out all the water [1]. Therefore, if the particle compression is negligible relative to the soil-mass compression, the two assumptive conditions concluded by Bishop and Blight [19] for effective stress equation are reasonable because of the wide existence of solid water which could affect both the particle compression and the inter-particle friction. The mechanism analysis in this section is the physical basis of the reasonability of these hypotheses.

\section{Discussion}

4.1. The Solid Water in the Ordinarily Dried Samples. It is important to confirm the definition of "absolutely dry" soil, because dried clays adsorb water from the atmosphere even at low relative humidity and temperatures, and even small amounts of adsorbed water could lubricate interparticle slip and reduce friction [10]. It is found that carefully dried slip surfaces had stronger frictional resistance than those exposed to room humidity [37]. It means that the "dry strength" may be a relative term depending on the length and temperature of drying, so Morrow et al. [33] carefully vacuum dried their "dry samples" at $180^{\circ} \mathrm{C}$ and sheared under vacuum. Otherwise, if samples are not dried and tested under vacuum, they may be just "nominally dry" samples with lower frictional strength than the "absolutely dry" ones [33].

However, in geotechnical engineering, most of conventional tests are conducted under ambient conditions but not vacuum, and samples are commonly not dried under vacuum too. When the ordinarily dried samples are taken out from an oven and exposed to ambient humidity, they could adsorb water molecules to form solid-water layer before to be tested. In other words, for conventional geotechnical tests under ambient conditions, the solid-water layer should always exist in soil samples during the whole process from 


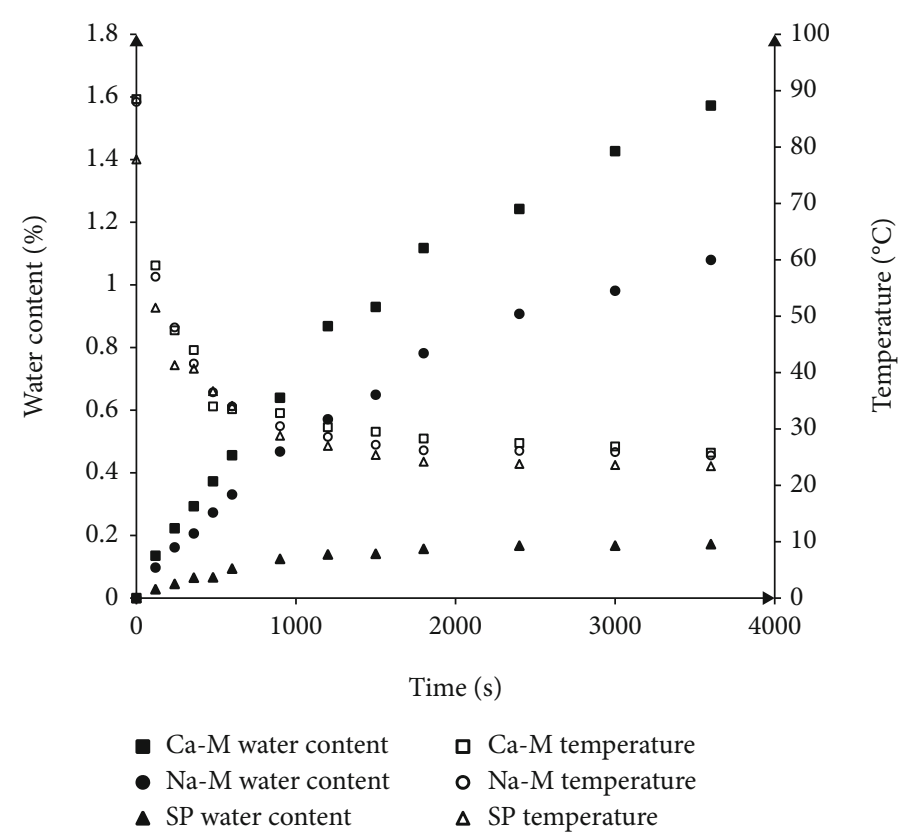

Figure 10: Water content and temperature as functions of time for oven dried Ca-montmorillonite (Ca-M), Na-montmorillonite (Na-M), and silica powder (SP) under ambient conditions.

they are taken out from an oven. A simple monitoring test on water content is conducted to certify such an assumption.

Three representative geomaterials, Ca-montmorillonite, Na-montmorillonite, and silica powder are used because montmorillonite is famous for its absorbability while silica is always considered less absorptive for comparison. For better reflecting the absorbability difference, silica powder is predried once for 24 hours and then situated under ambient conditions for 24 hours, to minimize its original water content. Therefore, Ca-montmorillonite, Na-montmorillonite, and silica powder have original water content as $15.44 \%$, $13.31 \%$, and $0.22 \%$, respectively. On a day with $22.4^{\circ} \mathrm{C}$ and $70.5 \% \mathrm{RH}$, three room dry samples are put into an oven to dry at $105^{\circ} \mathrm{C}$ for 48 hours. Afterwards on a day with $23.3^{\circ} \mathrm{C}$ and $59.9 \% \mathrm{RH}$, samples are taken out from the oven then to continually monitor their water contents and temperatures in 1 hour. Test data are plotted in Figure 10.

It is clearly shown in Figure 10 that all samples immediately start to adsorb water molecule in atmosphere from they are taken out from the oven, so the water content is increasing with the decreasing temperature. After 1 hour, the water contents of Ca-montmorillonite and Na-montmorillonite increase to $1.57 \%$ and $1.08 \%$, respectively. Meanwhile even for the silica powder, its water content recovers to $0.17 \%$ which is close to the original water content $0.22 \%$.

Based on the test results, it is concluded that for the ordinarily oven-dried soil samples used in conventional geotechnical tests carried out under ambient conditions but not vacuum, they are just "nominally dry" samples which could adsorb water molecules from the atmosphere. Therefore, the test data should have reflected the lubrication due to the solid-water layer that always exists in the whole test process.

4.2. The Effects of Solid Water on the Shear Strength of Soils. If soil particles are commonly wrapped by solid-water layer

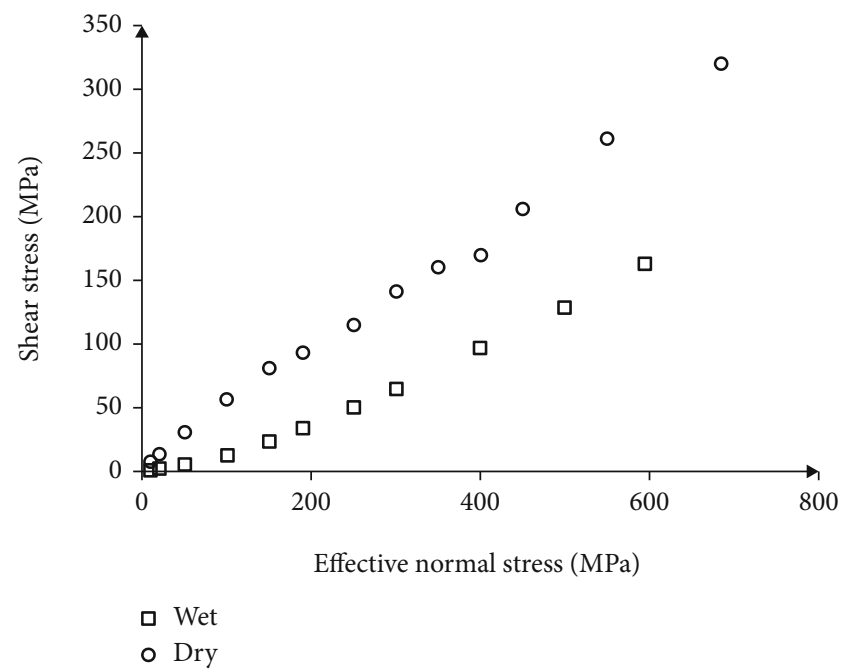

FIGURE 11: Shear stress as a function of effective normal stress for dry and wet samples (replotted with selected data in Figure 5: (a) of Reference [10]).

under ambient conditions, i.e., they contact each other indirectly; perhaps, the conventional test results have involved the lubrication effect of solid water, especially for the clays with strong adsorption ability. For some tests with rigid boundary such as in Figure 9, the interparticle solid-water layer is possible to be squeezed out during test process, but for some clays with strong adsorption ability, perhaps a large effective stress is needed to squeeze solid-water layer out. However, it is found that even the effective normal stress is increased to $600 \mathrm{MPa}$, the wet friction strength is still about half of the dry friction strength [10], as shown in Figure 11.

Figure 11 clearly shows that the wet friction strength is always lower than the dry friction strength even though the 
effective normal stress is increased to $600 \mathrm{MPa}$. Considering other conditions are consistent in both tests, the only reason for the strength difference should be the existence of water in samples. Moreover, Morrow et al. [10] designed a sample geometry and test sequence that would keep internal pore pressure in equilibrium with the external control system and carefully conducted these tests slowly enough to avoid pore fluid overpressure, so the influence of liquid water which could flow as normal on the strength could be limited while the solid-water layer should play an important role to reduce the strength of wet samples due to its lubricity. It means that the solid-water layer wrapping soil particles has profound effect on the friction strength of adsorptive clays. If the solid-water layer exists from the beginning of a test, even though some inter-particle solid water in some instantaneous force chains could be extruded, other solid-water layer on particle surface could affect the reconstruction of force chains during the whole test process. Especially in highly saturated condition, the extruded interparticle solid water could also recover quickly when the contact points separate in the reconstruction process of force chains. It could be the reason why the wet friction strength is always lower than the dry friction strength.

\section{Conclusion}

After a brief review on the properties of adsorbed water, a sixphase model for soils is introduced to define solid water. Then, the mechanisms how the solid-water layer affects the particle compression and the interparticle friction are analyzed based on simple models to clarify the physical basis of the reasonability of two assumptive conditions for effective stress.

In conclusion, for particle skeletons with absorptivity (hydrophilism), the solid-water layer should widely exist in soils under ambient conditions. If the interparticle solidwater layer could be extruded due to self-weight of particles or external loads with suitable confinement, or evaporated by high temperature, the particles could really contact with each other ("particle-particle" contact), so there is no interparticle lubrication due to solid water and particles directly exert inter-particle forces to each other. In this case, if the water pressure is increased, the particles could be compressed more and the interparticle stiction/friction should be enhanced. Otherwise, if the interparticle solid-water layer always exists, i.e., the interparticle contact is the "lubricated contact" ("particle-solid water-particle" contact); the increasing water pressure could induce just more particle compression. Moreover, particles indirectly affect each other via the hydration force, which means that because of the "lubricated contact," force chains are in the configuration of "particlesolid water-particle" so the load in chains is transmitted among particles via hydration force.

Finally, for a soil mass without external load, the particles should be suspended in water because the solid water layer is not likely to be extruded by pressure alone; therefore, if the particle compression is negligible relative to the soil-mass compression, the two assumptive conditions for effective stress equation are reasonable.
In fact, real effects relating to solid water are much more complicated; many confused problems should be considered in the future, such as how to real timely monitor the amount of solid water during the test process and how to confirm the existence of inter-particle solid water during the reformation process of force chains in soils with different saturation.

\section{Data Availability}

The water-content monitoring-test data used to support the findings of this study are available from the corresponding author upon request.

\section{Conflicts of Interest}

The author declares there are no conflicts of interest regarding the publication of this paper.

\section{Acknowledgments}

The author gratefully acknowledges the China Scholarship Council (CSC) for the sponsorship.

\section{References}

[1] J. K. Mitchell and K. Soga, Fundamentals of Soil Behavior, Wiley, Hoboken, 2005.

[2] T. K. Tokunaga, "Hydraulic properties of adsorbed water films in unsaturated porous media," Water Resource Research, vol. 45 , no. $6,2009$.

[3] N. Lu and M. Khorshidi, "Mechanisms for soil-water retention and hysteresis at high suction range," Journal of Geotechnical and Geoenvironmental Enginneering, vol. 141, no. 8, article 04015032, 2015.

[4] M. Khorshidi, N. Lu, and A. Khorshidi, "Intrinsic relationship between matric potential and cation hydration," Vadose Zone Journal, vol. 15, no. 11, pp. 1-12, 2016.

[5] M. Tuller, D. Or, and L. M. Dudley, "Adsorption and capillary condensation in porous media: liquid retention and interfacial configurations in angular pores," Water Resource Research, vol. 35, no. 7, pp. 1949-1964, 1999.

[6] M. Tuller and D. Or, "Water films and scaling of soil characteristic curves at low water contents," Water Resource Research, vol. 41, no. 9, 2005.

[7] T. P. Leão and M. Tuller, "Relating soil specific surface area, water film thickness, and water vapor adsorption," Water Resource Research, vol. 50, no. 10, pp. 7873-7885, 2014.

[8] M. E. Chenevert, "Shale alteration by water adsorption," Journal of Petroleum Technology, vol. 22, no. 9, pp. 1141-1148, 1970.

[9] N. Miura and T. Yamanouchi, "Effect of water on the behavior of a quartz-rich sand under high stresses," Soils and Foundations, vol. 15, no. 4, pp. 23-34, 1975.

[10] C. A. Morrow, D. E. Moore, and D. A. Lockner, "Frictional strength of wet and dry montmorillonite," Journal of Geophysical Research: Solid Earth, vol. 122, no. 5, pp. 3392-3409, 2017.

[11] P. F. Low, "The clay/water interface and its role in the environment," Progress in Colloid \& Polymer Science, vol. 95, pp. 98107, 1994.

[12] R. Prost, T. Koutit, A. Benchara, and E. Huard, "Sate and location of water adsorbed on clay minerals: consequences of the 
hydration and swelling-shrinkage phenomena," Clays and Clay Minerals, vol. 46, no. 2, pp. 117-131, 1998.

[13] N. Lu and Y. Dong, "Correlation between soil shrinkage curve and water-retention characteristics," Journal of Geotechnical and Geoenvironmental Engineering, vol. 143, no. 9, article 4017054, 2017.

[14] D. G. Fredlund and N. R. Morgenstern, "Stress state variables for unsaturated soils," Journal of Geotechnical and Geoenvironmental Engineering, vol. 103, no. GT5, article ASCE 12919, 1977.

[15] D. G. Fredlund and H. Rahardjo, Soil Mechanics for Unsaturated Soils, Wiley, New York, 1993.

[16] V. I. Osipov, "The 2012 Hans Cloos lecture: physicochemical theory of effective stress in soils," Bulletin of Engineering Geology and the Environment, vol. 73, no. 4, pp. 903-915, 2014.

[17] Z. Zhang and X. Cheng, "Effective stress in saturated soil: a granular solid hydrodynamics approach," Granular Matter, vol. 16, no. 5, pp. 761-769, 2014.

[18] K. Terzaghi, Theoretical Soil Mechanics, Wiley, New York, 1943.

[19] A. W. Bishop and G. E. Blight, "Some aspects of effective stress in saturated and partly saturated soils," Géotechnique, vol. 13, no. 3, pp. 177-197, 1963.

[20] A. W. Skempton, "Effective stress in soils, concrete and rocks," in Selected Papers on Soil Mechanics, pp. 106-118, Thomas Telford, London, 1984.

[21] V. I. Osipov, "Nanofilms of adsorbed water in clay: mechanism of formation and properties," Water Resources, vol. 39, no. 7, pp. 709-721, 2012.

[22] P. F. Low, "Physical chemistry of clay-water interaction," Advances in Agronomy, vol. 13, pp. 269-327, 1961.

[23] R. T. Martin, "Adsorbed water on clay: a review," Clays and Clay Minerals, pp. 28-70, 1962.

[24] J. Hu, X. D. Xiao, D. F. Ogletree, and M. Salmeron, "Imaging the condensation and evaporation of molecularly thin films of water with nanometer resolution," Science, vol. 268, no. 5208, pp. 267-269, 1995.

[25] P. B. Miranda, L. Xu, Y. R. Shen, and M. Salmeron, "Icelike water monolayer adsorbed on mica at room temperature," Physical Review Letters, vol. 81, no. 26, pp. 5876-5879, 1998.

[26] N. Dhopatkar, A. P. Defante, and A. Dhinojwala, "Ice-like water supports hydration forces and eases sliding friction," Science Advances, vol. 2, no. 8, article e1600763, 2016.

[27] S. Leikin, V. A. Parsegian, D. C. Rau, and R. P. Rand, "Hydration forces," Annual Review of Physical Chemistry, vol. 44, no. 1, pp. 369-395, 1993.

[28] C. Zhang, "Hydration force," in Encyclopedia of Tribology, Springer, Boston, 2013.

[29] J. N. Israelachvili and R. M. Pashley, "Molecular layering of water at surfaces and origin of repulsive hydration forces," Nature, vol. 306, no. 5940, pp. 249-250, 1983.

[30] J. N. Israelachvili and P. M. McGuiggan, "Forces between surfaces in liquids," Science, vol. 241, no. 4867, pp. 795-800, 1988.

[31] U. Raviv and J. Klein, "Fluidity of bound hydration layers," Science, vol. 297, no. 5586, pp. 1540-1543, 2002.

[32] J. N. Israelachvili, P. M. McGuiggan, and A. M. Homola, "Dynamic properties of molecularly thin liquid films," Science, vol. 240, no. 4849, pp. 189-191, 1988.

[33] C. A. Morrow, D. E. Moore, and D. A. Lockner, "The effect of mineral bond strength and adsorbed water on fault gouge fric- tional strength," Geophysical Research Letters, vol. 27, no. 6, pp. 815-818, 2000.

[34] T. Saarenketo, "Electrical properties of water in clay and silty soils," Journal, vol. 40, no. 1, pp. 73-88, 1998.

[35] X. Duan, L. Zeng, and X. Sun, "Generalized stress framework for unsaturated soil: demonstration and discussion," Acta Geotechnica, vol. 14, no. 5, pp. 1459-1481, 2019.

[36] V. I. Osipov, Physicochemical Theory of Effective Stress in Soils, Springer, New York, 2015.

[37] J. H. Dieterich and G. Conrad, "Effect of humidity on timeand velocity-dependent friction in rocks," Journalof Geophysical Research: Solid Earth, vol. 89, no. B6, pp. 4196-4202, 1984. 